\title{
Analysis of blood flow and local expression of angiogenesis-associated growth factors in infected wounds treated with negative pressure wound therapy
}

\author{
CHENG-YAN XIA, AI-XI YU, BAIWEN QI, MIN ZHOU, ZONG-HUAN LI and WEI-YANG WANG \\ Department of Micro-Orthopaedics, Zhongnan Hospital of Wuhan University, Wuhan, Hubei 430071, P.R. China
}

Received August 5, 2013; Accepted February 19, 2014

DOI: $10.3892 / \mathrm{mmr} .2014 .1997$

\begin{abstract}
Angiogenesis is involved in the wound healing process. Increased angiogenesis and blood flow constitute a major mechanism of negative pressure wound therapy (NPWT), which has been shown to facilitate the healing of infected wounds. However, the effect on the expression of angiogensis-related growth factor remains unknown. The goal of the current study was to investigate the angiogenic factor levels prior to and following NPWT in infected wounds. A total of 20 patients with infected wounds treated with NPWT were included in the study. Patients acted as their own control; the postoperative measurements of patients were considered as the experimental group, while preoperative measurements were considered as the controlled group. Blood flow was recorded prior to and during NPWT. A total of 10 angiogensis-related growth factors were detected using a protein biochip array to analyze the change in protein levels prior to NPWT, and on the third day during NPWT. All wounds were successfully reconstructed by skin grafting or using local flaps following NPWT. NPWT resulted in significantly increased blood flow in the wound. There was a significant increase in vascular endothelial growth factor (VEGF), EGF, platelet-derived growth factor and angiotesin-2 following NPWT, while basic fibroblast growth factor decreased significantly. NPWT affects the local expression of angiogenesis-associated growth factors, which represents another mechanism to explain how NPWT accelerates wound healing.
\end{abstract}

\section{Introduction}

Wound healing and the management of difficult-to-treat wounds have been the focus of traumatology departments,

Correspondence to: $\mathrm{Dr} \mathrm{Ai}-\mathrm{Xi} \mathrm{Yu}$, Department of Micro-Orthopaedics, Zhongnan Hospital of Wuhan University, 169 East Lake Road, Wuhan, Hubei 430071, P.R. China

E-mail: yuaixi666@163.com

Key words: negative pressure wound therapy, angiogenesis-associated growth factors, protein biochip array, blood flow, infected wound particularly the infected wound. It has been well recognized that negative pressure wound therapy (NPWT) accelerates wound healing. The NPWT device included a section of foam placed in the wound and covered with an occlusive dressing (1). The functions of NPWT include resolving infection, completing wound debridement, reducing the time required to produce a healthy granulation bed and increasing wound contraction (2). Although extensive research has been performed to investigate the mechanisms of action by which NPWT increases the rate of healing, and its probable beneficial effects in various basic and clinical circumstances, the molecular mechanism of NPWT has rarely been reported to interpret this phenomenon

A crucial link of how the NPWT system promotes wound healing mainly focuses on the increased blood supply. Angiogenesis is a complex process, which is controlled and regulated by angiopoietin and anti-angiogenesis factors. Various growth factors, including vascular endothelial growth factor (VEGF), fibroblast growth factors (acidic and basic FGFs) and angiopoietin are involved in neovascularization (3). The angiogenesis-associated growth factors that essentially contribute to the maturation, stabilization and remodeling of the vasculature were hypothesized to be differentially expressed following treatment with a NPWT system in an infected wound.

In order to detect the expression of these angiogenesis-associated growth factors, a protein biochip array technique was used, which was constructed on the surface of a biochip. A carrier component transports the biochips to different treatment stations within the analyzer, and following ligand binding, a chemiluminescent signal is produced, which is measured by a charge-coupled camera device and quantified by imaging software (4). To the best of our knowledge, this is the first study in which a protein biochip array technology has been used to detect the molecular mechanism of the NPWT system.

\section{Materials and methods}

Patients. A total of 20 patients (13 males and 7 females) aged between 28 and 47 years (mean, 36.4) with a full-thickness infected wound, which could not be closed immediately at the Department of Orthopedics in Zhongnan Hospital of Wuhan University (Wuhan, China), were include in this study. Patients consented to partaking in the study. The etiology of all wounds was trauma. All patients required initial debridement and 

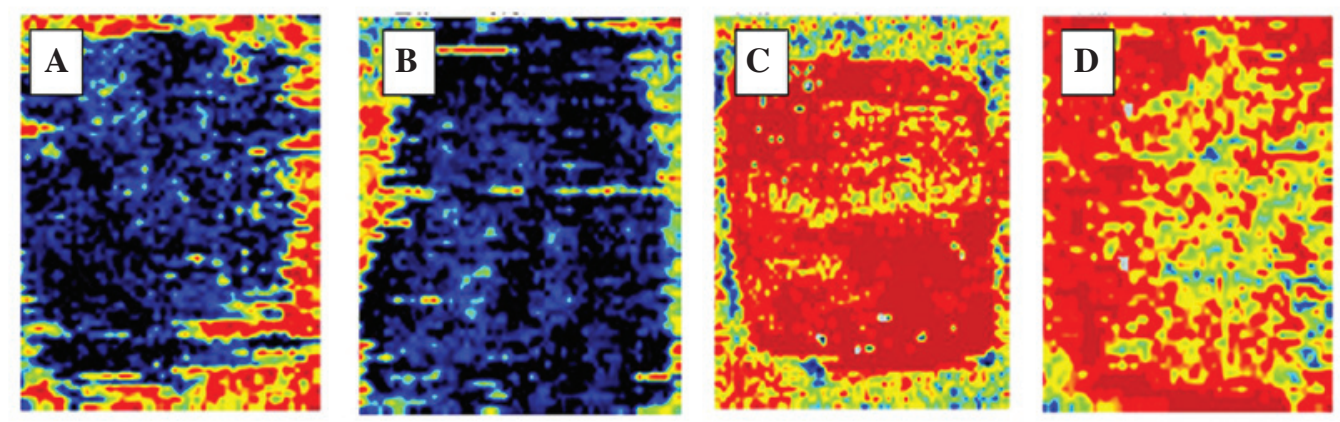

Figure 1. There were four common samples of all images of wound blood flow. The areas and shade of red in the image represents the quantity of blood flow in the wound, while the blue areas imply less or no blood flow. (A) Blood flow image of a traumatic wound prior to NPWT. (B) Blood flow image of a foot wound caused by trauma with the stress hyperglycemia initiated prior to NPWT. (C) A traumatic wound on the third day during NPWT. (D) A foot wound caused by trauma the third day during NPWT. Less blood flow was detected prior to NPWT and a marked increase occurred following NPWT. NPWT, negative pressure wound therapy.

necrotic soft tissue excisions. Spaced holes were drilled in the exposed bone and necrotic exposed tendons were excised. Following a proper debridement, patients were administered with a NPWT foam under a continuous pressure of $-125 \mathrm{mmHg}$ (all materials from VSD Medical Technology Co.,Ltd., Wuhan, China), no layer of protection was used between the foam and the wound. Various antibiotics, according to a drug sensitivity test, were used until the infection had been controlled. All wounds were finally covered following a number of VSD dressing renewals when a clean, red granulating wound bed was achieved. A full-thickness skin graft or a local transposition flap was selected as a final covering based on the situation of granulation tissue and exposed deep tissue. The study was approved by the local ethical committee (approval no. 2011059, Zhongnan Hospital of Wuhan University, Wuhan, China).

Following primary debridement and on the third day during NPWT, a Laser Doppler Blood Perfusion imager (Perimed Ltd., Beijing, China) and PeriScan PIM 3 system (Perimed Ltd.) was used to scan the blood flow of each wound, and the PIMsoft software (version 1.5; (Perimed Ltd.) was used to analyze the blood flow on the scanned images. At the same time, a $3 \times 5 \times 5 \mathrm{~mm}$ granulation tissues sample in the centre of each wound was collected. Then all samples were maintained at $-80^{\circ} \mathrm{C}$ until analyzed.

Proteomic analysis. Quantbody human angiogenesis antibody array 1 (RayBiotech Inc., Norcross, GA, USA) was used to assay the samples mentioned previously, 10 angiogenesis-associated growth factors, including angiogenin, angiotesin-2 (ANG-2), EGF, heparin-binding EGF-like growth factor (HB-EGF), leptin, bFGF, placenta growth factor (PIGF), VEGF, platelet-derived growth factor (PDGF) and hepatocyte growth factor (HGF) were detected in the current study. Samples of granulation tissues were treated consulting the specification of biochip array used. The protein array was performed following the user manual (version July 2010).

Statistical analysis. Results are expressed as the mean \pm standard deviation and were compared by analysis of variance. Values of each angiogenesis factor expression level and blood flows pre-primary debridement and three days later were compared. Data were analyzed with the SPSS 17.0 (SPSS. Inc., Chicago, IL, USA) software. $\mathrm{P}<0.05$ was considered to indicate a statistically significant difference.

\section{Results}

Outcomes of wound repair. No complications were noted regarding NPWT. Following NPWT, all patients presented with a clean, red, granulating wound bed and successful reconstruction had been achieved by skin grafting (15 patients) or using local flaps (5 patients). The average wound healing time was 31 days (range, 21-44 days). The number of dressing changes was between 1 and 4 , with an average of 1.8 times.

Wound blood flow. All wounds were scanned using the PeriScan PIM 3 system, and an image of each wound was captured (Fig. 1). A mean quantitative value of blood flow in the whole wound was calculated by PIMsoft software (Table I). A marked boost of blood flow was observed following NPWT, the mean blood flow prior to NPWT was $63.38 \pm 15.09$; and on the third day this increased statistically to $297.13 \pm 54.67$ during NPWT $(\mathrm{P}<0.001)$.

Protein biochip array analysis. The granulation tissue samples collected prior to and on the third day during NPWT, were observed to detect the changes in the expression level of the factors mentioned on the biochip array. Examples of the array are shown in Fig. 2. When angiogenesis factors were compared pre- and during NPWT therapy, an increase was observed in the protein levels of angiogenin, EGF, ANG-2, hepatocyte growth factor (HGF), PDGF, VEGF and PIGF; however, significant variations only occurred in ANG-2 $(\mathrm{P}=0.001)$, EGF $(\mathrm{P}=0.000)$, PDGF $(\mathrm{P}=0.018)$ and VEGF $(\mathrm{P}=0.000)$. In addition, the levels of bFGF significantly decreased $(\mathrm{P}=0.007)$. The expression levels of HB-EGF and leptin were too low to measure the variation following NPWT (Fig. 3).

\section{Discussion}

A complex soft tissue defect wound is difficult to manage. Application of NPWT has been shown to exhibit a marked 
Table I. The records of blood flow of each wound $(n=20)$ prior to and following NPWT.

\begin{tabular}{|c|c|c|c|}
\hline Patient & Wound area, $\mathrm{cm}$ & $\begin{array}{c}\text { Mean blood flow } \\
\text { of wound prior to NPWT }\end{array}$ & $\begin{array}{l}\text { Mean blood flow of wound } \\
\text { on the third day during NPWT }\end{array}$ \\
\hline 1 & $3 \times 5 \times 6$ & 50.6 & 231.9 \\
\hline 2 & $4 \times 6 \times 9$ & 68.7 & 322.7 \\
\hline 3 & $6 \times 7 \times 9$ & 88.3 & 309.9 \\
\hline 4 & $7 \times 8 \times 9$ & 34.5 & 289.5 \\
\hline 5 & $4 \times 6 \times 7$ & 56.7 & 356.7 \\
\hline 6 & $7 \times 7 \times 9$ & 55.2 & 332.6 \\
\hline 7 & $2 \times 4 \times 8$ & 76.8 & 347.2 \\
\hline 8 & $5 \times 7 \times 8$ & 88.5 & 198.6 \\
\hline 9 & $7 \times 8 \times 8$ & 56.7 & 334.7 \\
\hline 10 & $6 \times 5 \times 5$ & 46.8 & 289.0 \\
\hline 11 & $4 \times 7 \times 10$ & 76.8 & 310.4 \\
\hline 12 & $7 \times 8 \times 8$ & 43.1 & 209.1 \\
\hline 13 & $8 \times 10 \times 12$ & 59.0 & 408.4 \\
\hline 14 & $3 \times 4 \times 5$ & 67.4 & 276.9 \\
\hline 15 & $4 \times 7 \times 9$ & 77.5 & 344.5 \\
\hline 16 & $5 \times 7 \times 8$ & 68.4 & 287.1 \\
\hline 17 & $3 \times 6 \times 9$ & 56.7 & 230.9 \\
\hline 18 & $4 \times 5 \times 11$ & 77.9 & 339.5 \\
\hline 19 & $5 \times 7 \times 10$ & 46.9 & 287.6 \\
\hline 20 & $4 \times 6 \times 6$ & 71.1 & 235.3 \\
\hline
\end{tabular}

Each patient exhibited a marked increase in blood flow in the wound; the difference was statistically significant $(\mathrm{P}<0.001)$. Mean \pm standard deviation is ${ }^{\mathrm{a}} 63.38 \pm 15.09$ and ${ }^{\mathrm{b}} 297.13 \pm 54.67$. NPWT, negative pressure wound therapy.

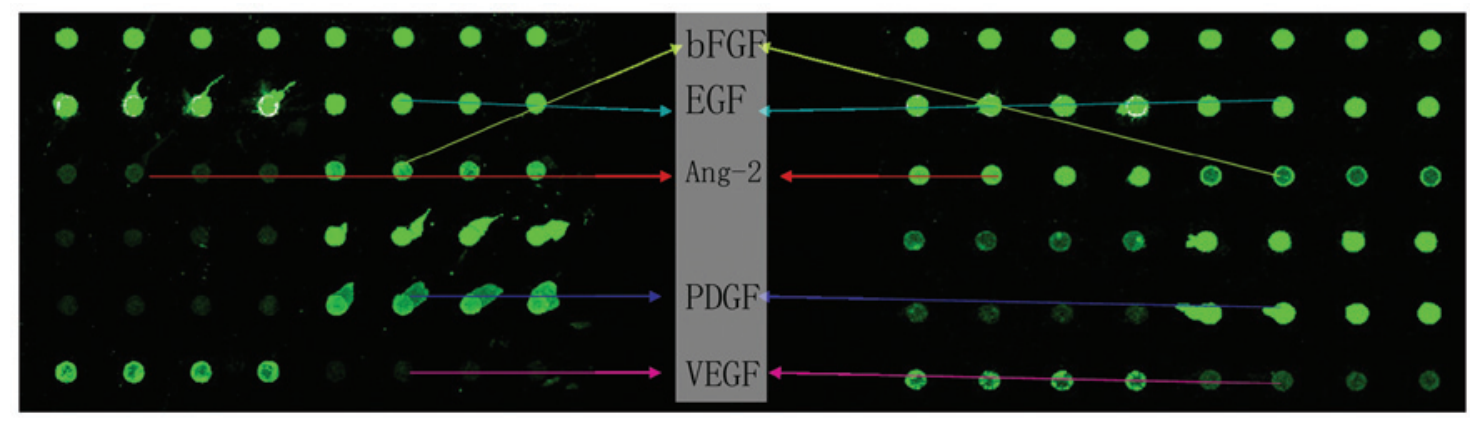

Figure 2. Scanned images of spectrum mean quantity indicate cytokines using a RayBio Biotin label-based Quantbody human angiogenesis antibody array 1 from granulation tissue samples prior to NPWT (left) and on the third day during NPWT (right). Intensity of every point is associated with the matching protein expression. Each four sequential points indicate a factor. All image points reflect the factors of POS1, POS2, angiogenin, ANG-2, EGF, bFGF, HB-EGF, HGF, leptin, PDGF, PIGF and VEGF from left to right and top to bottom, while the two factors topside were reference points. NPWT, negative pressure wound therapy; ANG-2 angiotesin-2; HB-EGF, heparin-binding EGF-like growth factor; HGF, hepatocyte growth factor; PDGF, platelet-derived growth factor; PIGF, placenta growth factor; VEGF, vascular endothelial growth factor.

effect on promoting wound healing (5). Earlier in vivo studies of wound healing showed that NPWT improved removal of edema fluid and increased blood flow, granulation tissue formation and bacterial clearance in wounds $(6,7)$. Lee et al (8) reported that NPWT application produces successful surgical reconstruction for large, deep skin and soft tissue defects without extensive radical flap surgery or loss of skin graft. In the current study, no complications were observed and high quality granulation tissue was achieved in each patient. Less local flaps were required during NPWT therapy, the wounds repaired using local flaps were one third of those that recieved skin grafting, and the success rate of the skin grafts were $100 \%$, due to the several advantages, including stabilizing the skin graft and preventing the collection of fluid under the skin graft (9).

The current study suggests that the increase of vasculogenesis and angiogenesis may be a significant effect of NPWT. Increased blood supply enhances the migration of inflammatory cells in and around the wound. Previous studies focus on the effect of angiogenesis-related factors during vasculogenesis 


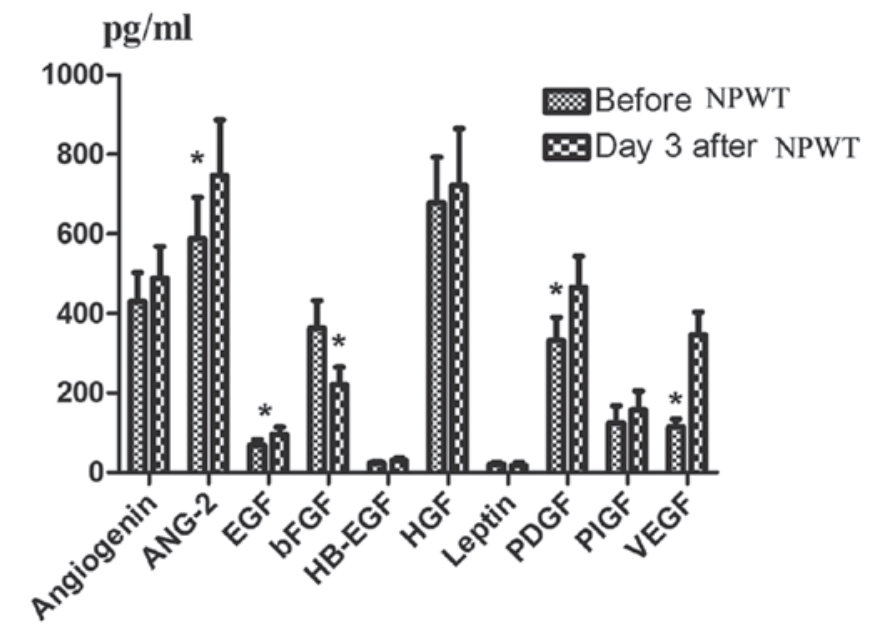

Figure 3. Angiogenic factors level prior to NPWT and on the third day during NPWT. Error bars represent the standard deviation, $\mathrm{n}=20$. ${ }^{*} \mathrm{P}<0.05$, compared with day 3 after NPWT. The level quantity of each factor and their change prior to and following NPWT. NPWT, negative pressure wound therapy.

and angiogenesis. These factors act on endothelial cells within blood vessels around the injured tissue leading to the sprouting of new capillaries to perfuse the wound tissue. These capillaries function to mobilize a diverse collection of cell types into the peripheral blood and promote angiogenesis, either by directly incorporating into neovasculature or indirectly by serving as an additional source of angiogenic growth factors/cytokines. In addition, they have been show to exhibit direct effects on keratinocytes $(10,11)$. Jacobs et al (12) used an in vivo application of the vacuum-assisted closure foam with negative pressure, which resulted in increased growth factor production, improved angiogenesis and collagen deposition. However, no previous study has used system to explore these cytokines following NPWT. The ability to detect a number of factors at once may have been problematic previously; however, protein chip detection has been broadly used in various fields (13) due to its advantages of high sensitivity, simultaneous detection of multiple protein expression levels, and ease of use. The use of a protein biochip array in the current study may provide a novel insight into molecular events, which may improve wound healing.

VEGF, a mitogenic factor, which acts on endothelial cells, is crucial in vasculogenesis and angiogenesis (14), and is regarded as one of the most important molecules involved in the process of angiogenesis (15). Labler et al (16) reported that the levels of VEGF in wound fluid were significantly higher in patients treated with NPWT compared with those treated with gauze. Our previous study showed an increase in VEGF levels using NPWT compared with a traditional gauze treatment (17). In addition, in this study significantly higher levels of VEGF were observed in the wound following NPWT.

ANG-2 is an angiogenic factor associated with maturation, stabilization and remodeling of vasculature (18), and promotion of endothelial cell survival (19). Shyu et al (20), observed a synergistic effect of angiopoietin and VEGF in promoting angiogenesis. This effect was implicated in the FOXC2-Ang2 signaling system, which is crucial for controlling vascular function (21). It has not been previously reported whether angiopoietin-2 levels alter following NPWT; however, in the current study, a significant increase was observed following NPWT.

PDGF is a co-factor that acts with VEGF to generate stable angiogenesis (22), and it has been reported to stimulate and enhance novel tissue formation in bone and soft tissue models (23). Combination of Ang-2 and PDGF may increase urokinase plasminogen-activator receptor expression (24), which is significant in the signaling pathways involved in wound healing. Shah et al (25) observed that the application of growth factors, such as PDGF, may aid in the healing of chronic, non-healing wounds. EGF is secreted by platelets, macrophages and fibroblasts, which act in a paracrine manner on keratinocytes (26). An in vitro study has shown that EGF was upregulated following acute injury, thus significantly accelerating reepithelialization (27). EGF and transforming growth factor $\beta$ (TGF- $\beta$ ) increase the tensile strength of the wound (28). $\mathrm{PlGF}$, an angiogenic mediator promoting pathophysiological neovascularization, is expressed during cutaneous wound healing (29). The diabetic mouse model of Erba et al (30) indicated that the NPWT device was associated with a significant activation of the wound, expression of PDGF and EGF, superior granulation tissue formation rich in collagen I, as well as superior wound epithelialization. Shah et al (25) found that the application of growth factors, including PDGF may aid in the healing of chronic, non-healing wounds. In the current study, all three cytokines were expressed at elevated levels following NPWT, however the increase of PIGF was not identified to be statistically significant. A study period longer than three days may demonstrate a significant increase in PIGF due to its involvement in improving in wound closure by enhancing angiogesis (29). A similar trend was observed with angiogenin, a plasma protein with angiogenic and ribonucleolytic activity implicated in wound healing. Further studies are required to investigate the lack of significant difference in the present study.

HGF is a multifunctional cytokine that is capable of stimulating multiple intracellular signaling pathways to induce a marked variety of biological activities in a wide spectrum of cell types. HGF stimulates migration and proliferation of keratinocytes and has been suggested to be involved in wound healing (31). Chen et al (32) also observed that HGF exhibits a potential role in reepithelialization and cilium hyperplasia. However, in the current study HGF levels were not identified to be significantly different following NPWT, however it remained at a high level and exhibited a rising tendency.

Leptin is a recently identified cell factor, which promotes novel angiogenesis, and modulates inflammatory and immune function (33). Murad et al (34) reported that leptin is acutely upregulated in the injured skin, and proposed that this local production of leptin serves a critical functional role as an autocrine/paracrine regulator of normal wound healing. A burn wound rat model suggested that topical application of leptin promotes reepithelization to shorten the wound healing time (35). An animal study by Kim et al (36) indicated that HB-EGF is involved in the wound-healing process. HB-EGF may reduce the required frequency of EGF application since it may cause prolonged EGFR signaling when immobilized in the wound (37). To the best of our knowledge, the current study was the first to demonstrate the influence of NPWT on leptin and HB-EGF; however, the results showed a low expression 
level and the changes were not significant. This may indicate that NPWT has less influence on leptin and HB-EGF.

As a single polypeptide, bFGF is one of the 22 members of the FGF family and is produced by a variety of cell populations, mainly by activated macrophages and thrombocytes (38). bFGF is involved in a number of physiological and pathophysiological processes, including growth, wound and bone healing, cell differentiation and proliferation. Local administration of bFGF in skin flaps markedly increased tissue viability and accelerated the wound healing process (39). Jacobs et al (12) designed an animal model in which NPWT led to increased expression of VEGF and bFGF in the first 5 days. However, in the current study, bFGF was observed at low levels on the third day during NPWT. A clinical phenomenon may indicate that incipient granulation tissue under NPWT easily collapses if the negative pressure is removed. The granulation tissue may lack collagen, which is not generated without bFGF. It perhaps performs at a higher level in the later phase during NPWT, thus, the tendency of bFGF under NPWT is hypothesized to increased following initial decrease. To the best of our knowledge, this is the first study to report this, thus, further studies are required to confirm this mechanism.

In addition, the angiogenesis-associated growth factors were not individually involved in angiogenesis, but interacted with and activated each other to promote angiogenesis. For example, in a study by Ko et al (40), VEGF enhanced neoangiogenesis, however, this was not effective on the maturation of organized reepithelization. The neoangiogenesis induced by EGF was not dominant, but did enhance the maturation of organized reepithelization. The key ability of bFGF is to induce angiogenesis via stimulation of VEGF expression (41). There are a number of studies of this, however these require further investigation. Investigation of the association and effects in disparate wounds and different phases may indicate whether angiogenesis modulation is involved in combined wound therapy.

The cause of these changes in the angiogenesis-associated growth factor levels and the underlying mechanisms were not mentioned in the current study. Quinn et al (42) showed that mechanical stretching regulates VEGF and bFGF gene expression in cultured pulmonary artery smooth muscle cells; however, the specific mechanisms remain unclear. The authors hypothesize that further research may provide an explanation. The factors detected in the current study are limited, as a number of other cytokines, including interleukin, TGF- $\beta$, insulin-like growth factor (IGF), GM-CSF, matrix metal proteinase 9 (MMP-9) are also involved in wound angiogenesis. Whether these cytokines are expressed differently under subatmospheric pressure also requires further investigation.

In conclusion, NPWT therapy can promote wound healing, and the mechanism may include: significantly increasing blood flows, and significant increases in VEGF, EGF, PDGF, ANG-2 and decreased bFGF after NPWT therapy. However, how these angiogenesis-associated growth factors act under subatmospheric pressure in wounds also requires further investigation.

\section{Acknowledgements}

This study was funded by grants from the National Science Funds of China (project code, 81171713), and by the Doctoral candidate Independent research of Wuhan University (grant no. 2012303020203).

\section{Reference}

1. Armstrong DG, Lavery LA, Abu-Rumman P, Espensen EH, Vazquez JR, Nixon BP and Boulton AJ: Outcomes of subatmospheric pressure dressing therapy on wounds of the diabetic foot. Ostomy Wound Manage 48: 64-68, 2002.

2. Argenta LC and Morykwas MJ: Vacuum-assisted closure: a new method for wound control and treatment: clinical experience. Ann Plas Surg 38: 563-577, 1997.

3. Murugesan S, Mousa SA, O'connor LJ, Lincoln DW II and Linhardt RJ: Carbon inhibits vascular endothelial growth factor- and fibroblast growth factor-promoted angiogenesis. FEBS Lett 581: 1157-1160, 2007.

4. Fitzgerald SP, Lamont JV, McConnell RI and Benchikh el O: Development of a high-throughput automated analyzer using biochip array technology. Clin Chem 51: 1165-1176, 2005.

5. de Laat EH, van den Boogaard MH, Spauwen PH, van Kuppevelt DH, van Goor H and Schoonhoven L: Faster wound healing with topical negative pressure therapy in difficult-to-heal wounds: a prospective randomized controlled trial. Ann Plast Surg 67: 626-631, 2011.

6. Morykwas MJ, Argenta LC, Shelton-Brown EI and McGuirt W: Vacuum-assisted closure: a new method for wound control and treatment: animal studies and basic foundation. Ann Plast Surg 38: 553-562, 1997.

7. Mouës CM, Vos MC, van den Bemd GJ, Stijnen T and Hovius SE: Bacterial load in relation to vacuum-assisted closure wound therapy: a prospective randomized trial. Wound Repair Regen 12: 11-17, 2004

8. Lee DL, Ryu AY and Rhee SC: Negative pressure wound therapy: an adjuvant to surgical reconstruction of large or difficult skin and soft tissue defects. Int Wound J 8: 406-411, 2011.

9. Hanasono MM and Skoracki RJ: Securing skin grafts to microvascular free flaps using the vacuum-assisted closure (VAC) device. Ann Plast Surg 58: 573-576, 2007.

10. Liu L, Marti GP, Wei X, Zhang X, Zhang H, Liu YV, Nastai M, Semenza GL and Harmon JW: Age-dependent impairment of HIF-1alpha expression in diabetic mice: Correction with electroporation-facilitated gene therapy increases wound healing, angiogenesis, and circulating angiogenic cells. J Cell Physiol 217: 319-327, 2008.

11. Wilgus TA, Matthies AM, Radek KA, Dovi JV, Burns AL, Shankar R and DiPietro LA: Novel function for vascular endothelial growth factor receptor-1 on epidermal keratinocytes. Am J Pathol 167: 1257-1266, 2005.

12. Jacobs S, Simhaee DA, Marsano A, Fomovsky GM, Niedt G and Wu JK: Efficacy and mechanisms of vacuum-assisted closure (VAC) therapy in promoting wound healing: a rodent model. J Plast Reconstr Aesthet Surg 62: 1331-1338, 2009.

13. Roesli C and Neri D: Methods for the identification of vascular markers in health and disease: from the bench to the clinic. J Proteomics 11: 2219-2229, 2010.

14. Ferrara N: Molecular and biological properties of vascular endothelial growth factor. J Mol Med (Berl) 77: 527-543, 1999.

15. Kadowaki I, Ichinohasama R, Harigae H, Ishizawa K, Okitsu Y, Kameoka J and Sasaki T: Accelerated lymphangiogenesis in malignant lymphoma: possible role of VEGF-A and VEGF-C. Br J Haematol 130: 869-877, 2005.

16. Labler L, Rancan M, Mica L, Härter L, Mihic-Probst D and Keel M: Vacuum-assisted closure therapy increases local interleukin-8 and vascular endothelial growth factor levels in traumatic wounds. J Trauma 3: 749-757, 2009.

17. Zhou M, Yu A, Wu G, Xia C, Hu X and Qi B: Role of different negative pressure values in the process of infected wounds healing treated by vacuum-assisted closure: an experimental study. Int Wound J 10: 508-515, 2013.

18. Maisonpierre PC, Suri C, Jones PF, et al: Angiopoietin-2, a natural antagonist for Tie 2 that disrupts in vivo angiogenesis. Science 277: 55-60, 1997.

19. Harfouche R and Hussain SN: Signaling and regulation of endothelial cell survival by angiopoietin-2. Am J Physiol Heart Circ Physiol 291: H1635-H1645, 2006.

20. Shyu KG, Chang $\mathrm{H}$ and Isner JM: Synergistic effect of angiopietin-1 and vascular endothelial growth factor on neoangiogenesis in hypercholesterolemic rabbit model with acute hindlimb ischemia. Life Sci 73: 563-579, 2003. 
21. Xue Y, Cao R, Nilsson D, et al: FOXC2 controls Ang-2 expression and modulates angiogenesis, vascular patterning, remodeling, and functions in adipose tissue. Proc Natl Acad Sci USA 105: 10167-10172, 2008.

22. Heldin CH and Westermark B: Mechanism of action and in vivo role of platelet-derived growth factor. Physiol Rev 79: 1283-1316, 1999.

23. Jin Q, Wei G, Lin Z, Sugai JV, Lynch SE, Ma PX and Giannobile WV: Nanofibrous scaffolds incorporating PDGF-BB microspheres induce chemokine expression and tissue neogenesis in vivo. PLoS One 3: e1729, 2008.

24. Bezuidenhout L, Bracher M, Davison G, Zilla P and Davies N Ang-2 and PDGF-BB cooperatively stimulate human peripheral blood monocyte fibrinolysis. J Leukoc Biol 81: 1496-1503, 2007.

25. Shah JM, Omar E, Pai DR and Sood S: Cellular events and biomarkers of wound healing. Indian J Plast Surg 45: 220-228, 2012

26. Shiraha H, Glading A, Gupta K and Wells A: IP-10 inhibits epidermal growth factor-induced motility by decreasing epidermal growth factor receptor-mediated calpain activity. J Cell Biol 146: 243-254, 1999.

27. Brown GL, Curtsinger L III, Brightwell JR, et al: Enhancement of epidermal regeneration by biosynthetic epidermal growth factor. J Exp Med 163: 1319-1324, 1986.

28. Brown GL, Curtsinger LJ, White M, et al: Acceleration of tensile strength of incisions treated with EGF and TGF-beta. Ann Surg 208: 788-794, 1998.

29. Cianfarani F, Zambruno G, Brogelli L, et al: Placenta growth factor in diabetic wound healing: altered expression and therapeutic potential. Am J Pathol 169: 1167-1182, 2006.

30. Erba P, Adini A, Demcheva M, Valeri CR and Orgill DP: Poly-N-acetyl glucosamine fibers are synergistic with vacuum-assisted closure in augmenting the healing response of diabetic mice. J Trauma 71: S187-S193, 2011

31. Nakamura T, Sakai K, Nakamura $T$ and Matsumoto $K$ : Hepatocyte growth factor twenty years on: Much more than a growth factor. J Gastroenterol Hepatol 26: 188-202, 2011.

32. Chen M, Guan M, Li J, Wang H and Yang B: Effects of hepatocyte growth factor on wound healing of rabbit maxillary sinus mucosa. J Otolaryngol Head Neck Surg 41: 253-258, 2012.
33. Goldberg AC, Goldberg-Eliaschewitz F, Sogayar MC, Genre J and Rizzo LV: Leptin and the immune response: an active player or an innocent bystander? Ann NY Acad Sci 1153: 184-192, 2009.

34. Murad A, Nath AK, Cha ST, Demir E, Flores-Riveros J and Sierra-Honigmann MR: Leptin is an autocrine/paracrine regulator of wound healing. FASEB J 17: 1895-1897, 2003.

35. Wen $\mathrm{H}, \mathrm{Wu} \mathrm{G}$, Chen W, Yang $\mathrm{H}$ and Fu J: Topical application of leptin promotes burn wound healing in rats. Nan Fang Yi Ke Da Xue Xue Bao 32: 703-706, 2012 (In Chinese).

36. Kim JM, Bak EJ, Chang JY, Kim ST, Park WS, Yoo YJ and Cha JH: Effects of HB-EGF and epiregulin on wound healing of gingival cells in vitro. Oral Dis 17: 785-793, 2011.

37. Tolino MA, Block ER and Klarlund JK: Brief treatment with heparin-binding EGF-like growth factor, but not with EGF, is sufficient to accelerate epithelial wound healing. Biochim Biophys Acta 1810: 875-878, 2011.

38. Robson MC, Phillips LG, Lawrence WT, et al: The safety and effect of topically applied recombinant basic fibroblast growth factor on the healing of chronic pressure sores. Ann Surg 216: 401-406, 1992.

39. Fayazzadeh E, Ahmadi SH, Rabbani S, Boroumand MA, Salavati A and Anvari MS: A comparative study of recombinant human basic fibroblast growth factor (bFGF) and erythropoietin $(\mathrm{EPO})$ in prevention of skin flap ischemic necrosis in rats. Arch Iran Med 15: 553-556, 2012.

40. Ko J, Jun H, Chung H, et al: Comparison of EGF with VEGF non-viral gene therapy for cutaneous wound healing of streptozotocin diabetic mice. Diabetes Metab J 35: 226-235, 2011.

41. Hayward P, Hokanson J, Heggers J, Fiddes J, Klingbeil C, Goeger $\mathrm{M}$ and Robson $\mathrm{M}$ : Fibroblast growth factor reserves the bacterial retardation of wound contraction. Am J Surg 163: 288-293, 1992

42. Quinn TP, Schlueter M, Soifer SJ and Gutierrez JA: Cyclic mechanical stretch induces VEGF and FGF-2 expression in pulmonary vascular smooth muscle cells. Am J Physiol Lung Cell Mol Physiol 282: L897-L903, 2002. 